\title{
El emprendimiento como fuente de desarrollo y fortalecimiento de las capacidades endógenas para el aprovechamiento de las energías renovables
}

Entrepreneurship as a source of development and strengthening of endagerous capacities for the utilization of renewable energy

L'entrepreuriat comme source de développement et d'amélioration des capacités endogènes pour l'utilisation des énergies renouvelables

Dempreendimento como fonte de desenvalvimento $\mathrm{E}$ fortalecimento das capacidades endógenas para $\square$ aproveitamento das energias renováveis
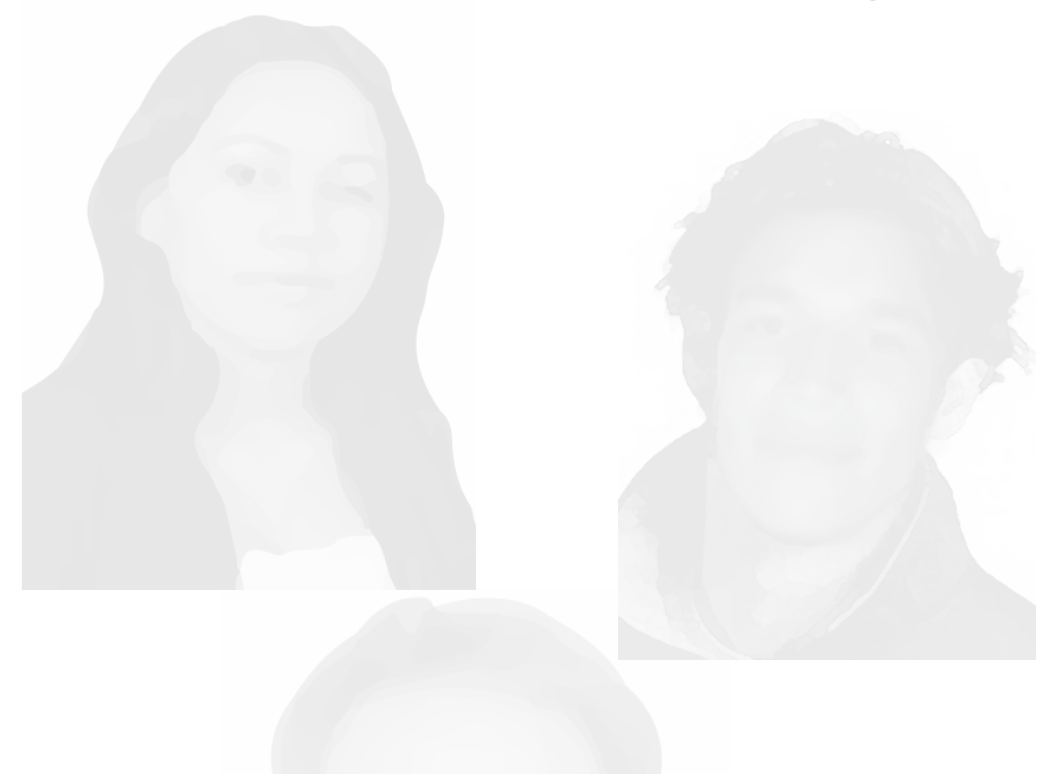

" Maestría en Administración Universidad Nacional de Colombia Contaduría Pública Universidad Nacional de Colombia

${ }^{* *}$ Especialización en Control Interno,Universidad Militar Nueva Granada. Administrador de Empresas, Universidad Militar Nueva Granada.

${ }^{* * *}$ Maestría en Administración, Especialización en Gerencia Integral de la Calidad Universidad Nacional de Colombia; Especialista en Docencia Universitaria, Universidad Militar Nueva Granada; Administrador de Empresas, Escuela de Administración de Negocios 


\section{RESUMEN}

Si bien muchas de las problemáticas ambientales actuales tienen un alcance global, sus impactos se experimentan a nivel local con repercusiones en los territorios, la población y la economía. Por tanto, las medidas para mitigar dichas problemáticas deben originarse en iniciativas locales que respondan de manera precisa a las necesidades identificadas por los actores sociales, a la vez que se haga uso de los recursos y las capacidades disponibles en el territorio.

Si en algo existe consenso, es en que hacer frente a los problemas ambientales actuales, demanda un cambio en los sistemas de producción y consumo, hacia prácticas que garanticen un uso más eficiente y sostenible de los recursos, en donde un cambio en el patrón energético juega un papel fundamental, debido al impacto que ha generado el uso de los combustibles fósiles. Frente a este desafío se requiere de la capacidad emprendedora e innovadora de los agentes, de manera que sea posible incorporar al sistema nuevos métodos de producción, nuevos insumos y productos, y nuevas formas de hacer las cosas.

Es en este sentido que las energías renovables cobran un papel importante dado que surgen como una alternativa para enfrentar el agotamiento de los combustibles fósiles y a su vez, como una forma de reducir las externalidades negativas asociadas al consumo de energía. Por tanto el objetivo del presente artículo, es presentar una revisión teórica respecto al papel que juega el emprendimiento en el desarrollo y fortalecimiento de las capacidades endógenas para el aprovechamiento local de las fuentes renovables de energía.

\section{ABSTRACT}

While current environmental problems are taken place worldwide, their impacts are seen in different regions, populations and economies. For this reason, all measures and plans to mitigate those problems should originate from local initiatives to respond to specific needs of social agents, making use of available resources and capacities in each territory.

Facing all these current environmental problems bring about changes in production and consumption systems which involve more efficient and sustainable use of resources, changing the energy pattern with its key role due to the impact in the use of non-renewable energies. To solve all these problems, an entrepreneurial and innovative capacity is needed to adopt new production methods, supplies and products, as well as new ways to do things.

As renewable energies play an important role as a new alternative to face the depletion of non-renewable energies and as a way to reduce the external negative factors associated with energy consumption. Then, this paper aims at showing a theoretical revision describing the relevance of entrepreneurship in the development and strengthening of endogenic capacities to take advantage of local renewable sources of energy.

\section{Palabras clave}

Capacidades endógenas Desarrollo endógeno y local Emprendimiento verde Energías renovables
Keywords

Endogenic capacities

Endogenic and local development

Green Entrepreneurship

Renewable and nonrenewable energies 


\section{RESUMÉÉ}

Bien que de nombreux enjeux environnementaux actuels aient une portée globale, leur impact et répercutions au niveau local sur la population et l'économie sont importants. Les mesures visant à atténuer ces problématiques devraient donner lieu à des initiatives locales répondant précisément aux besoins identifiés par les acteurs sociaux et utiliser les ressources disponibles sur le territoire.

Il existe de nos jours un consensus pour résoudre les problèmes environnementaux actuels qui exigent une évolution, un changement dans les systèmes de production et de consommation vers des pratiques qui assurent une utilisation plus efficiente et durable des ressources. La modification de la configuration de l'énergie joue un rôle clé dans ce processus en raison de l'impact généré par l'utilisation des combustibles fossiles. Pour faire face à ce défi, les capacités d'innovation et d'entrepreneuriat deviennent essentielles pour qu'il soit possible d'intégrer de nouvelles méthodes de production, de nouveaux intrants, de nouveaux produits et de nouvelles façons de concevoir les choses.

Voilà comment les énergies renouvelables jouent un rôle considérables car elles représentent une alternative pour remédier à l'épuisement des stocks d'énergies fossiles et sont en même temps un moyen de réduire les externalités négatives associées à la consommation d'énergie. Voilà pourquoi l'objectif de ce document est de présenter un examen théorique montrant le rôle joué par l'entrepreneuriat dans le développement et le renforcement des capacités endogènes d'utilisation locale des sources d'énergie renouvelables.

\section{RESUMO}

Se bem muitas das problemáticas ambientais atuais têm alcance global, seus impactos são experimentados a nível local com efeitos nos territórios, a população e a economia. Portanto, as medidas para mitigar essas problemáticas devem ser originadas em iniciativas locais que respondam com precisão às necessidades identificadas pelos atores sociais, e que ao mesmo tempo se faça uso dos recursos e as capacidades disponiveis no território.

Se há algo em que todos coincidem, é que enfrentar os problemas ambientais atuais, requere mudanças nos sistemas de produção e consumo, em direção a práticas que garantam um uso mais eficiente e sustentável dos recursos, onde uma mudança no padrão energético tem um papel fundamental, devido ao impacto gerado pelo uso dos combustiveis fósseis. Diante este desafio precisa-se da capacidade empreendedora e inovadora dos agentes, de maneira que seja possivel incorporar ao sistema novos métodos de produção, novos insumos e produtos e novas formas de fazer as coisas.

É neste sentido que as energias renováveis têm um papel importante dado que surgem como uma alternativa para enfrentar o esgotamento dos combustíveis fósseis e ao mesmo tempo, como una maneira de reduzir as externalidades negativas relacionadas com o consumo de energia. Portanto, o objetivo deste documento é apresentar uma revisão teórica respeito ao papel do empreendimento no desenvolvimento e fortalecimento das capacidades endógenas para o aproveitamento local das fontes renováveis de energia.

\section{Mots clefs}
Développementocal et endogène
Capacités endogènes
Energie renouvelable
Entrepreneuriat vert

\section{Palavras-chave}

Capacidades endógenas Desenvolvimento endógeno e local Empreendimento verde Energias renováveis 


\section{INTRODUCCIÓN}

$\mathbf{L}$ as crisis ambientales y algunas crisis económicas que se han venido registrando en las últimas décadas, como las crisis del petróleo ${ }^{10}$ y del cambio climático ${ }^{11}$ con sus múltiples efectos, por mencionar algunas de ellas, han dejado entrever la inadecuada gestión que se ha realizado de los recursos naturales bajo el sistema económico imperante y la fuerte dependencia de las economías de los combustibles

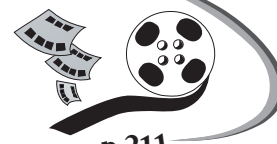
fósiles. Estos procesos se han generado como consecuencia de un crecimiento económico que no tiene en cuenta los límites, especialmente por parte de las naciones industrializadas.

Lo anterior, poniendo en riesgo la base de los recursos naturales que soportan las actividades presentes y futuras y la subsistencia misma del ser humano (MAVDT, 2010). A su vez, tales crisis controvierten algunos supuestos del modelo capitalista y ponen sobre la mesa algunos debates importantes, como la disponibilidad limitada de los recursos naturales, la capacidad restringida de carga del planeta, la interrelación e interdependencia de los sistemas naturales y sociales y la dicotomía existente entre ambiente y desarrollo (MAVDT, 2010; Sabogal, 2009).

Bajo este contexto, se hace inminentemente necesario un cambio en los patrones de producción y consumo hacia prácticas que garanticen un uso más eficiente de los recursos naturales y disminuyan los impactos negativos multidimensionales de la interacción del ser humano con su entorno. Dicho cambio en los patrones mencionados, implica modificar la dieta en consumo de recursos, generación de desechos y emisiones de Gases Efecto Invernadero (GEI).
En este sentido, en las últimas décadas se ha prestado una creciente atención al aprovechamiento de las fuentes de energías renovables, en parte, bajo el argumento de que necesita hallarse una solución a lo que ha sido connotado como un problema de "sostenibilidad ambiental", y también, debido al requerimiento cada vez más apremiante de sustituir el uso de combustibles fósiles por fuentes renovables e ilimitadas de energía. Lo mencionado, a fin de disminuir la dependencia energética de las naciones y prepararse para lo que ha sido denominado como el fin del petróleo barato, consecuencia de la disminución de su disponibilidad y la dificultad para explotar las fuentes atípicas del mismo (Coderch, 2004; citado en Ballenilla, 2005); ello, teniendo en cuenta que es precisamente la energía la base del actual modelo capitalista (Páez, 2002).

Por tanto, las políticas centradas en la promoción del uso de las fuentes de energías renovables, pueden ser consideradas una de las variables que encuadra en la compleja ecuación que conduce a la mitigación de las problemáticas ambientales y de disponibilidad y seguridad energética actuales. Adicionalmente, dado que su aprovechamiento depende de la dotación de recursos disponibles a nivel local en cada territorio, estas se consideran además una alternativa para promover el desarrollo de las comunidades locales, generando impactos positivos sobre el empleo, la calidad del ambiente, el desarrollo tecnológico y la innovación y la organización social, entre otros aspectos, vía el surgimiento de iniciativas emprendedoras que además de aprovechar las capacidades locales, conduzcan a lograr las metas cada vez más ambiciosas de incorporación de las energías limpias en las canastas energéticas.

\footnotetext{
${ }^{10}$ Entre ellas, la denominada primera crisis del petróleo ocurrida en 1973 por el embargo de la Organización de Países Exportadores de Petróleo que impidió el suministro de petróleo a los países que habían apoyado a Israel durante la guerra de Yom Kippur.

11 “Un cambio de clima atribuido directa o indirectamente a la actividad humana que altera la composición de la atmósfera mundial y que se suma a la variabilidad natural del clima observada durante periodos de tiempo comparables" (Naciones Unidas, 1992).
} 
Teniendo en cuenta lo anterior, el objetivo del presente trabajo, es resaltar el papel que juega el emprendimiento en torno a la creación y el fortalecimiento de capacidades endógenas para el aprovechamiento de las fuentes de energías renovables. Para lograr el objetivo mencionado, este documento aborda en la primera parte la concepción de capacidades endógenas y desarrollo local, posteriormente hace referencia al papel que juegan las energías renovables en el marco de la sostenibilidad y cómo el emprendimiento es origen y consecuencia de la creación y fortalecimiento de las capacidades endógenas, para finalmente exponer algunos retos que debe enfrentar Colombia para generar desarrollo endógeno y local a partir del aprovechamiento de las fuentes renovables de energía.

Las reflexiones que aquí se presentan, se derivan del desarrollo de la primera etapa del proyecto de investigación denominado "Capacidades endógenas en el desarrollo e implementación de energías renovables: el caso de Bogotá" adelantado por el grupo de investigación en Ciencias Económicas (CIE) de la Universidad Militar Nueva Granada. Dichas reflexiones fueron allegadas a partir de la revisión y el análisis teórico en torno a temas tales como: desarrollo endógeno y local, energías renovables y emprendimiento. Adicionalmente, se indagó sobre experiencias de implementación de proyectos de energías renovables en Colombia.

\section{LAS CAPACIDADES ENDÓGENAS EN EL NÚCLEO DEL DESARROLLO LOCAL}

1 unque pueda ser visto como algo contradictorio, es en el actual mundo globalizado interconectado e interdependiente, en donde el desarrollo local del territorio ha retomado una importancia significativa y creciente, debido a la dificultad para que desde las aras del mercado global se coordinen soluciones frente a los problemas específicos de cada rincón del planeta, enmarcados en un sistema político, social, económico, institucional y cultural particular constituyendo lo que fue denominado por Madoery (2008; citado en Furlan, 2010) como la diversidad de realidades territoriales.

Es por ello que ante las crisis sociales, económicas y financieras, con sus múltiples efectos sobre el empleo, la disponibilidad de recursos, la salud, el desarrollo humano, la calidad de vida de la población, entre otros, se hace evidente una evolución hacia nuevas formas de organización de la actividad económica y social, en donde las acciones se empiezan a gestar desde los ámbitos locales, como respuesta a las necesidades inmediatas que atañen a los actores sociales (Sarría, 2008; Vázquez, 2009), reafirmándose la idea que la capacidad creativa e innovadora emerge con más ahínco, en tiempos de crisis (Vázquez, 2009).

De hecho, como lo plantea Boisier (2005) cuando se habla de desarrollo, el término en sí mismo ya quiere decir desarrollo local, endógeno y humano. Lo anterior debido a que cuando se hace referencia a un país desarrollado, no se podría generalizar esta condición a toda la población presente en su territorio, afirmando que la misma se encuentra en igualdad de condiciones ya que no sería así. En este sentido, para evaluar el nivel de desarrollo de una comunidad se debe hacer una aproximación individual con relación a sus dinámicas sociales, ambientales y económicas, en lo que puede ser aludido como geografías del desarrollo.

Si bien el enfoque de desarrollo local ha sido considerado principalmente desde una óptica económica y ha sido analizado como la respuesta de los países en desarrollo a fin de neutralizar los efectos negativos de la globalización y así lograr el ajuste productivo, un aspecto al que se ha prestado menor atención ha 
sido a su papel frente a la construcción de capital social (Gallicchio, 2004), entendido como "las normas, redes y organizaciones con las que la gente accede al poder y a los recursos, y a través de los cuales se toman decisiones colectivas y se formulan las políticas" (Barreriro, citado en Gallicchio, 2004) en un marco de interacción social a través de la cooperación y la asociación.

Así mismo, el desarrollo local se visualiza hoy como una forma de hacer frente a otro tipo de problemáticas, como aquellas de tipo ambiental que si bien en algunos casos tienen una connotación global, deben ser contrarrestadas a partir de acciones locales, dado que tienen efectos directos sobre la población local y se manifiestan en forma distinta en cada territorio, entre ellos: la contaminación, la desertificación, el cambio climático, la disponibilidad de agua, entre otros.

En este sentido el desarrollo local puede ser definido desde la óptica de uno de los más notables exponentes del "nuevo regionalismo" europeo, Garofoli (1995, citado en Boisier, 2005) como:

"la capacidad para transformar el sistema socio-económico; la habilidad para reaccionar a los desafíos externos; la promoción de aprendizaje social, y la habilidad para introducir formas específicas de regulación social a nivel local que favorecen el desarrollo de las características anteriores. Desarrollo endógeno es, en otras palabras, la habilidad para innovar a nivel local".

De otra parte, la Comisión Económica para América Latina CEPAL (citada en Sarría, 2008) define desarrollo local como:

...un proceso de crecimiento y cambio estructural que mediante la utilización del potencial de desarrollo existente en el territorio conduce a la mejora del bienestar de la población de una localidad o una región. Cuando la comunidad local es capaz de liderar el proceso de cambio estructural, la forma de desarrollo se puede convenir en denominarla desarrollo local endógeno.
Gallicchio (2004) por su parte, lo define como un proceso de concertación entre los actores sociales presentes en un territorio, orientado a desarrollar un proyecto común de desarrollo que implica la generación de crecimiento económico, equidad, cambio social y cultural, sustentabilidad ecológica, calidad y equilibrio espacial y territorial, a fin de elevar la calidad de vida de la población vinculada al territorio, a la vez que se contribuye al desarrollo de la nación y se enfrentan los retos y transformaciones del ambiente externo.

Bajo esta óptica, el territorio puede ser entendido como una agrupación de relaciones mercantiles y sociales, que se conjugan con diferentes formas de regulación social, determinando así las diferentes maneras de organizar la producción y de generar innovación (Garofoli, 1992; citado en Furió, 1994). En palabras de Madoery (2008 p141), lo local es un ámbito de construcción de poder, de control, de dominación, de hegemonías, de conflictos, pero también de acuerdos.

Desde el enfoque de desarrollo local existen dos aspectos que condicionan los resultados de las acciones que se planteen; de un lado, el potencial de desarrollo existente en el territorio representado en los recursos disponibles y la capacidad del recurso humano; y del otro, las capacidades de organización de los actores sociales para lograr acciones coordinadas. En palabras de Vázquez (2009) y Romero (2009) cada localidad, país o territorio posee una determinada estructura productiva, mercado de trabajo, conocimientos técnicos, capacidad empresarial, recursos naturales, estructura social y política, o tradición y cultura, sobre los que necesariamente se articulan las iniciativas locales. Según Furió (1994), el éxito o fracaso del funcionamiento de una economía local depende cada vez más de las variables endógenas inherentes al territorio, por lo que el mismo se convierte en un factor estratégico de desarrollo y definidor de sus características.

En este sentido, las capacidades endógenas de desarrollo pueden ser definidas como la dotación de aptitudes, talentos y cualidades de las cuales dispone un territorio para el aprovechamiento de sus recursos y potencialidades y que se configuran a partir de aspectos estructurales, institucionales y relacionales presentes en el mismo (Furlan, 2010). 
Dentro de las iniciativas que se considera conllevan a un desarrollo local humano, íntegro y duradero, se encuentran entre otras: el fortalecimiento de la estructura empresarial existente en el territorio y el fomento de la capacidad emprendedora y de gestión de los actores sociales; la incorporación, aprendizaje y difusión de conocimiento, innovaciones y tecnología, integrado a una política de desarrollo del capital humano o lo que denomina Alburquerque (1999; citado en Gallicchio, 2004), la construcción de entornos innovadores; el desarrollo de una infraestructura adecuada y la conservación y mejora del ambiente y del patrimonio histórico y cultural de la región, junto a la coordinación de los diferentes programas y proyectos que se diseñen para promover el desarrollo (Gallicchio, 2004; Vázquez, 2009).

Es necesario aclarar que el desarrollo local no implica un desarrollo desligado y desarticulado de las dinámicas nacionales, regionales y globales, sino un desarrollo que si bien está inmerso en estas dinámicas, es planificado y gestionado desde el ámbito local, desde un enfoque de abajo-arriba, coordinando las acciones públicas con los intereses locales y capitalizando al máximo las capacidades disponibles en el territorio (Sarría, 2008). Como lo menciona Bervejillo (1999), es una combinación simultánea de desterritorialización y reterritorialización, en donde por un lado hay un surgimiento de sistemas globales y por otro, un retorno al territorio como factor clave de desarrollo y de decisión (Gallicchio, 2004).

En palabras de Silva-Lara (2005 p83; citado en Arias y Villalta, 2009).

Sobre todo en el plano territorial, es cada vez más importante diseñar instrumentos y políticas públicas de gestión dirigidas a estimular el aprovechamiento de los recursos locales endógenos para impulsar nuevos estilos de desarrollo basados en las potencialidades de las economías locales, como complemento indispensable de las políticas nacionales de desarrollo.

En términos de Gallicchio (2004), el desarrollo local debe ser un desarrollo multidimensional e integrador, que busca armonizar lo local con lo global y que debe ser gestado poractores de desarrollo, quienes deben orientarse hacia la negociación y la cooperación, en búsqueda de una transferencia y fortalecimiento de capacidades y no solo de recursos. Para ello, deben enfrentarse tres desafíos: potenciar lo existente en el territorio, obtener recursos externos al territorio fortaleciendo las redes de apoyo y colaboración y gestionar el excedente generado. Lo anterior, a fin de pasar de una actitud pasiva en donde el desarrollo local ha sido asimilado a las ayudas económicas de índole pública a través de subsidios a la población vulnerable, al compromiso activo e involucramiento de las comunidades desde una perspectiva de solidaridad, innovación y crecimiento (Alburquerque, 2001; Boisier, 2005). Por tanto, lo que se pretende es un adecuado trabajo en equipo entre el Estado, el mercado y los demás actores locales, prevaleciendo el trabajo en redes y no las jerarquías, la influencia y no la autoridad (Gallicchio, 2004).

Así, el desarrollo endógeno puede verse reflejado en diferentes ámbitos: en lo político como la capacidad para influir en el desarrollo y formulación de políticas, en el ámbito financiero, como la capacidad para conciliar los intereses a largo plazo de la comunidad con los intereses de los inversionistas externos y en el ámbito cultural y tecnológico, como el desarrollo sin perder de vista los orígenes y el legado de las anteriores generaciones (Boisier, 2005). En palabras de Sarría (2008), en esta concepción del desarrollo el punto principal de referencia es el territorio, el potencial endógeno, la cercanía a los problemas y a quienes los sufren, y la movilización, la cooperación y la solidaridad.

Con relación a la adaptación del modelo de desarrollo endógeno en América Latina, Gudynas (2011) afirma que es una economía sustentada aún en el extractivismo y concluye que bajo estas condiciones el "desarrollo endógeno" se pierde, debido a que no se posee autonomía frente a los mercados globales. Bajo este estilo de desarrollo, el empleo generado es insuficiente, la productividad es suplantada por mayores volúmenes exportados y la presión sobre los recursos naturales aumen-

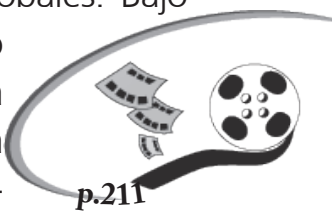
ta, y con ello, los conflictos sociales. Es un modelo sustentado en la creencia que las exportaciones y la explotación de los recursos naturales son la solución a los problemas de desigualdad y pobreza, frente a lo que plantea que sigue siendo necesario generar estrategias de desarrollo endógenas y autónomas. 


\section{RESPUESTAS LOCALES A LAS PROBLEMÁTICAS AMBIENTALES ACTUALES}

S bien algunas problemáticas ambientales hacen presencia a nivel global, sus repercusiones y efectos se experimentan en forma diferente a nivel local, como es el caso del cambio climático, frente a lo cual, las acciones deben planearse y coordinarse desde el ámbito local como respuesta a las necesidades propias de cada territorio. Como lo manifiesta Canoura (2010), el cambio en los patrones de producción y consumo requerido para contrarrestar las problemáticas ambientales actuales, no podría llegar a buen término si las decisiones a nivel de los gobiernos no se acompañan con cambios en la conducta de las comunidades. Incluso, las comunidades pueden y deben influir en las políticas públicas, a través de la generación de experiencias y aprendizajes mediante el desarrollo de acciones de gestión ambiental a nivel local.

En este sentido, el desarrollo local puede conjugarse con los aspectos ambientales a través de la definición de desarrollo sostenible local, el cual es definido por Carrizosa (2005, citado en Sabogal y Moreno, 2011) como:

Dependiente de las particularidades del lugar, se sujeta a las condiciones, los cambios y la creatividad para el uso del territorio por sus habitantes, está definida a través de procesos, se hace visible en la interacción de la sostenibilidad biofísica con la sostenibilidad cultural del lugar, incorpora la participación de todos los estamentos de la sociedad y el alcance de las decisiones políticas, atendiendo a valores éticos de equidad intra -e intergeneracional (Carrizosa, 2005, p36).

Según Sabogal y Moreno (2011), el desarrollo sostenible alude entonces, al proceso que asegura para un grupo poblacional, sociedad o comunidad, lograr el mejoramiento de sus condiciones de vida mientras mantiene las condiciones biofísicas y ecológicas críticas, que son esenciales para la sobrevivencia colectiva. En este sentido, el abordaje alternativo viene soportado en la planificación biorregional (Guimarâes, 2001; citado en Sabogal y Moreno, 2011) y la visión de desarrollo a diferentes escalas territoriales, asumiendo la desigualdad en el desempeño de los territorios y acciones que abarcan lo social, lo ambiental y lo económico (Borja \& Castells, 1998; Carmona Maya, 2001; Carrizosa, 2001; Clark, 1995; Escobar, 1996; Moncayo, 2001; Sagar, 2000; citados en Sabogal y Moreno 2011). En términos de Unda (2005, citado en Larrea, 2010), la sostenibilidad implica producir diferente, consumir diferente y organizarse diferente. 


\section{LAS ENERGÍAS RENOVABLES EN EL MARCO DEL DESARROLLO SOSTENIBLE LOCAL}

$\gamma_{y}$ omo respuesta a las problemáticas económicas y ambientales actuales, en las últimas décadas se ha prestado una creciente atención al aprovechamiento de las fuentes de energías renovables, en parte, bajo el argumento de que necesita hallarse una solución a lo que ha sido connotado como un problema de "sostenibilidad ambiental", el cual puede definirse como una externalidad negativa, resultado de la acumulación de los efectos adversos generados por las actividades humanas durante siglos. A este respecto, se considera que las energías renovables permitirán generar

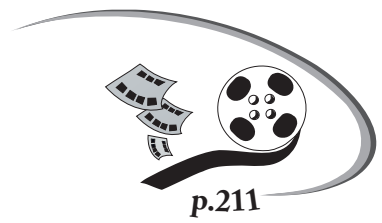
un cambio en los patrones actuales de producción y consumo, ya que parte de los daños ambientales causados dependen de los tipos de fuentes de energía utilizados y de las tecnologías que se emplean para suministrar los artículos y servicios que la sociedad necesita (BBVA, 2010).

Otra de las justificaciones que ha apalancado el interés por dichas fuentes de energías, es el requerimiento cada vez más apremiante de sustituir el uso de combustibles fósiles por fuentes renovables e ilimitadas de energía. Lo mencionado, a fin de disminuir la dependencia energética de las naciones y prepararse para lo que ha sido denominado como el fin del petróleo barato, consecuencia de la disminución de su disponibilidad y la dificultad para explotar las fuentes atípicas del mismo (Coderch, 2004; citado en Ballenilla, 2005), ello, teniendo en cuenta que es precisamente la energía la base del actual modelo capitalista (Páez, 2002).

En este sentido, las políticas centradas en la promoción del uso de las fuentes de energías renovables, pueden ser consideradas una de las variables que encuadra en la compleja ecuación que conduce a la mitigación de la problemáticas ambientales y de disponibilidad y seguridad energética actuales. Por lo anterior, no es aventurado pensar que estamos en la era de la transición energética que se caracteriza por una pérdida de la participación relativa de los hidrocarburos y un aumento gradual de las energías alternativas y de las convencionales revalorizadas (Furlan, 2010), lo que se espera conduzca a nuevas y más abundantes fuentes de energía (Páez, 2002).

No obstante, se originan varios cuestionamientos en torno a las energías renovables que deben ser abordados y que limitan el surgimiento de iniciativas emprendedoras alrededor de las mismas, dentro de ellas, las condiciones desequilibradas del mercado emergente de las energías renovables, en el cual estas tienen que competir con energías tradicionales que no internalizan los costos ambientales y que por tanto son menos costosas, frente a lo que se requiere en un entorno regulatorio adecuado (Sáenz de Miera, 2007). No obstante, López (2013) afirma que se espera que con el tiempo, el costo de las energías renovables entre en descenso conforme se agotan las fuentes fósiles de energía y a su vez avanza el desarrollo tecnológico del sector.

Otro aspecto que debe tenerse en cuenta respecto al aprovechamiento de las fuentes renovables de energía, son los gastos en que debe incurrirse para el desarrollo de la nueva tecnología y la transformación de la infraestructura, los artefactos y la cultura para su adaptación efectiva. Así mismo, debe evaluarse la tasa de retorno energético de dichas fuentes de energía, a través de la cual se mide la cantidad de energía invertida en obtener energía, pues fuentes renovables como el hidrógeno y el etanol consumen más energía en el proceso de obtención que la que aportan. Máxime cuando el insumo que se utiliza para la producción de los mismos es el petróleo (Ballenilla, 2005).

Finalmente, se deben enfrentar cuestiones como el hecho de que si bien las energías renovables pueden entrar a suplir algunas necesidades asociadas al transporte, la industria y el consumo energético de 
los hogares, no son un sustituto perfecto del petróleo dado que no podrán sustituir los insumos utilizados por la industria petroquímica, los cuales son la base de la producción de diversos artículos de uso cotidiano como artículos deportivos, juguetes, envases y empaques, materiales de construcción, muebles y enseres del hogar, zapatos, televisores, radios, computadoras y toda clase de artículos eléctricos y electrónicos cuyo insumo es el plástico y los hules derivados del petróleo, además de emplearse para cubrir las necesidades primordiales del ser humano, vestido, salud y alimentación. Además como lo afirma Yáñez-Arancibia et al (2010) la mayoría de las "energías alternativas" experimentadas son de desempeño y eficiencia pobre debido a su reciente desarrollo.

Pese a lo anterior, las tecnologías renovables están experimentando una progresiva tasa de crecimiento en todo el mundo, al tiempo que los objetivos de desarrollo definidos con relación a ellas a medio plazo, son cada vez más ambiciosos, lo que puede ser explicado a partir de las ventajas que tienen respecto a tres ámbitos fundamentales: el ambiente, la seguridad energética y el desarrollo económico.

Desde el punto de vista ambiental, las energías renovables se renuevan de forma natural, por lo que pueden ser consideradas infinitas, no producen gases de efecto invernadero y son, por tanto, una de las pocas vías disponibles para hacer frente al aumento de la demanda de energía sin agravar el problema del cambio climático. Desde la perspectiva energética, se trata de energías autóctonas, que no es necesario importar, están disponibles en mayor o menor medida en todos los países y contribuyen, por tanto, a reducir la elevada y creciente dependencia de la mayor parte de los países. Por último, las energías renovables son una importante fuente de empleo y constituyen, en muchos casos y especialmente en regiones poco avanzadas, un motor de desarrollo económico y social (Sáenz de Miera, 2007).

En este sentido, se considera que las energías renovables son una alternativa que permite un modelo de desarrollo limpio ambientalmente, territorialmente descentralizado y autogestionado a diferentes niveles (Pérez, et al, 2005), permitiendo además, la autosuficiencia de las regiones respecto a la disponibilidad de energía (Páez, 2002). No obstante para que ello suceda se requiere de un entorno propicio para su desarrollo, como lo afirma Sáenz de Miera (2007), las energías renovables no se instalan como en principio podría pensarse, donde más y mejores recursos naturales hay sol, viento, agua, ni en los países más grandes, sino donde mejores marcos regulatorios existen. Lo anterior no implica necesariamente apoyos económicos sino más bien reglas de juego predecibles, estables y mejor adaptadas a las necesidades de unas inversiones como las que requieren las energías renovables, muy intensivas en capital y a largo plazo.

Por tanto, bajo este nuevo contexto de necesidades, se plantea el desarrollo de instrumentos sociales para la gestión energética relacionados con la educación y la participación social, como consecuencia de la existencia de evidencia que gran parte de la falta de éxito de algunas políticas se deriva de la falta de implicación de las poblaciones a las que se dirigen (Pérez, et al, 2005).

En este sentido, se considera que los actores locales pueden asumir un papel significativo, asimilando una gestión integrada y participada de la energía, a través del desarrollo de políticas, como por ejemplo, enfocadas en la promoción del emprendimiento de energías alternativas a nivel local. Lo que además contribuiría a energizar asentamientos poblacionales de las zonas no interconectadas a las redes eléctricas nacionales. También inspiraría aprendizajes valiosísimos para la construcción de los futuros modelos energéticos territoriales, es decir, basados en las capacidades endógenas y en los recursos locales. Así mismo, la implementación de programas activos de educación energética puede trabajarse eficazmente en la escala local (Furlan, 2010).

A nivel empírico, una experiencia recopilada en una región de Argentina, pero que es reflejo de la situación de otros países de América Latina, hace evidente que con relación a la planeación energética actual, el modelo de desarrollo más aplicado es de tipo centralizado de arribaabajo y localmente impuesto desde el exterior, esto es, se deriva de macro programas diseñados para una nación, desde los cuales se determina la planificación de suministros, el nivel requerido de inversiones, la fijación 
de precios de mercado, que prescinde de actuaciones promovidas desde el ámbito local. Bajo esa concepción, la disponibilidad de energía a nivel local es considerada simplemente como un factor garantizado de forma externa y por tanto, parte del inventario de recursos del territorio, esto es, no existe apropiación del proceso de su generación (Furlan, 2010).

Lo mencionado debido al poder que han ejercido los monopolios en el suministro de energía, particularmente en algunos eslabones de la cadena productiva, principalmente en la distribución, ejerciendo el control de la oferta de energía y de los precios, manipulando de esta forma la matriz energética de las naciones e impidiendo incluso el desarrollo de las energías renovables. En este sentido, podría afirmarse que los agentes del mercado eléctrico actúan favoreciendo sus intereses enfocados en la apropiación de la renta energética, promoviendo incluso, si es necesario, el subdesarrollo eléctrico (Furlan, 2010).

Lo anterior parece contradictorio con la idea de promover el desarrollo endógeno alrededor del aprovechamiento de las fuentes alternativas de energía, por ende, para que tenga sentido en los sistemas culturales, la energía debe ser encauzada, dirigida y gobernada (White, 1982 p341; citado en Furlan, 2010); por tanto puede ser considerada condición y al mismo tiempo, objeto de desarrollo endógeno.
En el caso europeo, en que sus áreas rurales se están despoblando con las subsecuentes consecuencias sobre la producción agrícola y el uso de los suelos, las energías renovables son consideradas como una alternativa para revertir este proceso, ofreciendo la posibilidad de que los campesinos se desempeñen en actividades alternativas o complementarias y de esta forma, generar empleo descentralizado como consecuencia de la dispersión de estas fuentes. Lo anterior teniendo en cuenta que las fuentes de energías renovables son más intensivas en trabajo que la producción de energía convencional para la misma cantidad de producción energética. No obstante, esto depende del tipo de energía de que se trate, pues por ejemplo la energía eólica si bien genera empleo, es temporal, mientras que el empleo generado en la generación de energía a través de biomasa, tiende a ser más permanente, lo que además debe balancearse con los costos de producción de dichas energías y su viabilidad de aprovechamiento (Burguillo y del Río, 2008).

Adicionalmente, se considera que las fuentes de energías renovables, al utilizar menos servicios y productos importados y al explotar principalmente los recursos locales, contribuye al desarrollo de la industria a nivel local y regional, estimulando la inversión local y el empleo (Burguillo y del Río, 2008). 


\section{EL EMPRENDIMIENTO INSULO Y PRODUCTO DEL DESARROLLO DE CAPACIDADES ENDÓGENAS PARA EL APROVECHAMIENTO DE LAS ENERGÍAS RENOVABLES}

E emprendimiento es considerado fuente de crecimiento económico, debido a que permite incorporar al sistema nuevos métodos de producción, nuevos insumos y productos y nuevas formas de hacer las cosas, promoviendo por tanto el progreso tecnológico, la innovación y la productividad (Lucas, 1990; Hall y Jones, 1999; citados en Valdiviezo, 2009). Por consiguiente, resulta importante para el desarrollo endógeno, la existencia de cualidades emprendedoras en los individuos que habitan el territorio que busca desarrollarse, de forma que aporten dinamismo (Formichella, 2004).

No obstante, el emprendimiento es insumo y a la vez producto del desarrollo de las capacidades endógenas de un territorio. De un lado, para que las iniciativas emprendedoras surjan, se requiere de la dotación de ciertas habilidades y talentos iníciales, en donde el contexto local proporciona los recursos y las relaciones necesarias para el desarrollo de la actividad productiva (Vázquez, 2005; citado en Valdiviezo, 2009), siendo entonces las características específicas del territorio las que promueven el surgimiento y el desarrollo de las empresas. Lo anterior dado que si bien, existen unas condiciones personales que caracterizan al empresario actual y potencial, como la motivación, el perfil, las fuentes de financiamiento, entre otras, el marco institucional y macroeconómico vigente en el sistema económico tiene una influencia decisiva sobre el tipo de actividades en las cuales ellos aplicarán sus capacidades y competencias (Valdiviezo, 2009).

De otra parte, el emprendimiento es fuente de aprendizaje y experiencia, lo que lleva a las empresas a modificar sus estrategias y acumular competencias, lo que a su vez permite el fortalecimiento y perfeccionamiento de dichas capacidades (Valdiviezo, 2009). En este sentido, existe una relación en doble vía entre emprendimiento y desarrollo endógeno, dado que se determinan y modifican mutuamente. En palabras de Formichella (2004), existe una retroalimentación entre el medio y las capacidades emprendedoras de los individuos.

Teniendo en cuenta que en palabras de Garofoli (1995, citado en Boisier, 2005) el desarrollo endógeno es la habilidad para innovar a nivel local, el emprendimiento se convierte entonces en una fuente importante de capacidades endógenas y un promotor del desarrollo local, mediante el aprovechamiento de los recursos disponibles en el territorio y la introducción y difusión de innovación y progreso tecnológico en el sistema productivo (Valdiviezo, 2009).

Con relación a las energías renovables, el emprendimiento constituye la vía a través de la cual se puede aprovechar el potencial de fuentes de energías renovables existentes en un territorio: radiación solar, viento, agua, entre otros; para la generación autóctona de energía, promoviendo la autosuficiencia en el suministro de la misma, contribuyendo a la sostenibilidad ambiental, el empleo y el desarrollo económico y social de las poblaciones, generando así esquemas de desarrollo local y endógeno. El emprendimiento representa además la posibilidad de no comprar tecnología desarrollada en el exterior con servicios de aplicación también importados Urquidi, 2009).

Lo anterior permite además, elevar el valor económico de las energías renovables, que por tratarse de un nuevo patrón genera empleos y promueve la reactivación de otras industrias de índole local, que pueden actuar como proveedores de aquella, pues hasta el momento han sido los proveedores internacionales los que se han beneficiado de este tipo de proyectos (Huacuz, citado en Urquidi, 2009). 
Además, por tratarse de una industria joven permite a las regiones ubicarse como pioneros en el desarrollo de nuevas tecnologías. Ello podría contribuir a reversar la tendencia observada en el mercado de las energías renovables, en el que América Latina figura como una de las regiones con mayor dotación de energías renovables, mientras que economías como Europa y América del Norte, figuran como las que mayor desarrollo tecnológico poseen para el aprovechamiento de las fuentes primarias (Huacuz, citado en Urquidi, 2009).

Dentro de los factores que se ha evidenciado, se han promovido el surgimiento de iniciativas emprendedoras alrededor del aprovechamiento de las fuentes de energías renovables entre las que se encuentran: marcos legales y regulación ad hoc, capacidades institucionales, incentivos oficiales para el desarrollo industrial, formación de recursos humanos y mecanismos financieros privados. Huacuz menciona que para el caso de México, se evidenció un marco legal reducido y un desarrollo institucional deficiente; a la vez que falta mucho financiamiento privado y público, y aunque se han evidenciado avances, aún falta formación profesional (Urquidi, 2009).

Lo anterior no difiere de la situación evidenciada en Colombia, en donde las políticas de carácter público se han enfocado en realizar estudios para determinar los potenciales de aprovechamiento de las fuentes renovables de energía, mostrando debilidades en el diseño de políticas para la generación endógena de conocimientos y tecnologías. No obstante, dado que la tendencia a nivel mundial en las próximas décadas se inclina hacia una mayor inversión en el sector eléctrico, respecto de la inversión en combustibles fósiles, emerge una gran posibilidad para desarrollar actividades emprendedoras e innovadoras en torno a las energías renovables, lo que implica además generar un ambiente local propicio para el desarrollo industrial, dado que en palabras de Huacuz hablar de energías renovables hoy, es hablar de compras del exterior (Urquidi, 2009). Se muestra los factores que inciden en la generación de un desarrollo endógeno a partir de las fuentes renovables de energías (Figura 1).

Figura 1. Factores que inciden en el desarrollo endógeno a partir del aprovechamiento de las fuentes renovables de energía

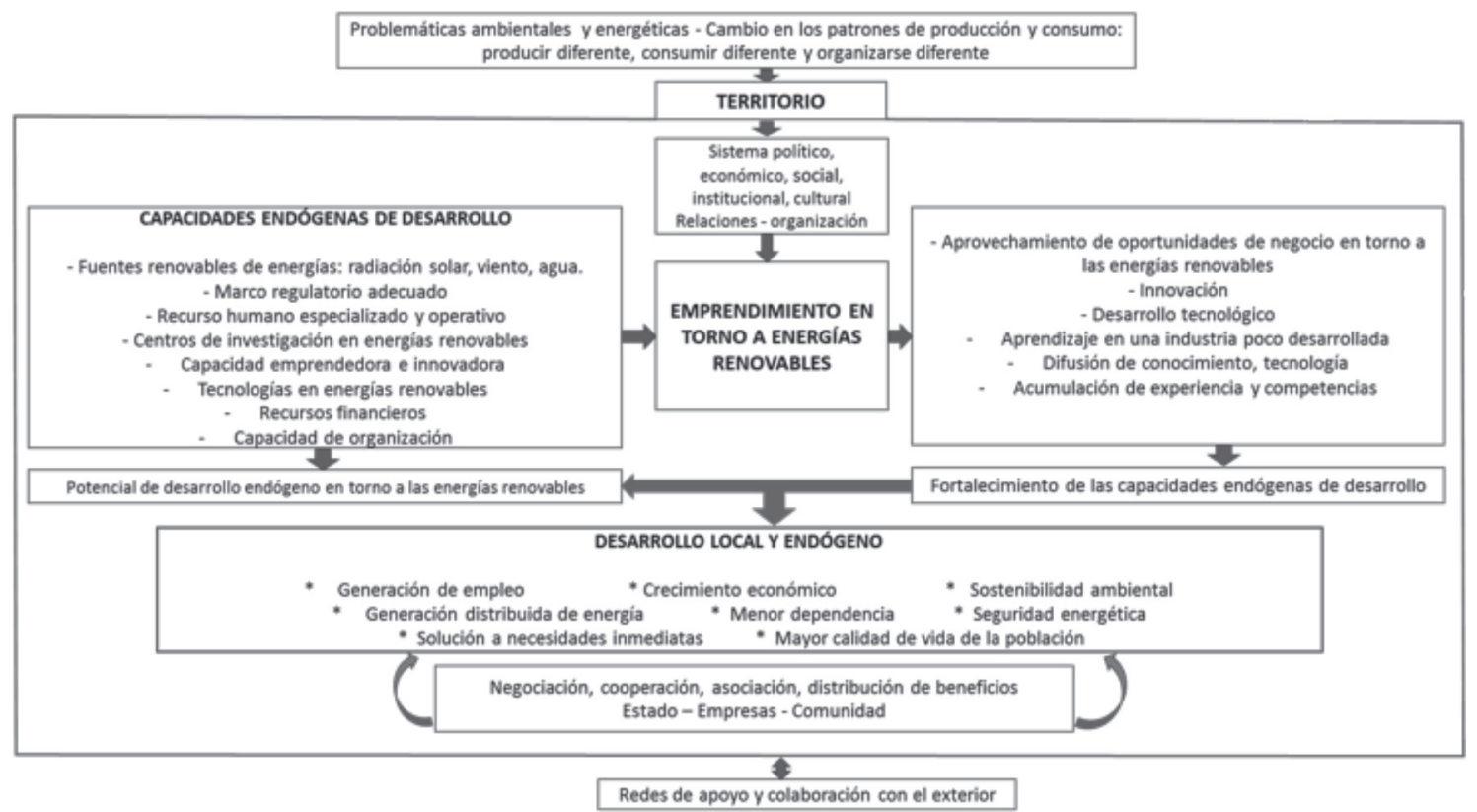

Fuente. Elaboración propia de los autores. 


\section{CONCLUSIONES}

L as fuentes renovables de energía se encuentran en mayor o menor medida disponibles en cada territorio, en recursos como la radiación solar, el viento, la disponibilidad de agua, entre otros, siendo América Latina considerada una de las regiones con mayor disponibilidad de ellas. No obstante, en la actualidad son los países de las regiones de Europa y América del Norte las que mayor desarrollo tecnológico han tenido para el aprovechamiento de las mismas, debido a su disponibilidad limitada de combustibles fósiles respecto de sus niveles de consumo y por tanto, la necesidad de reducir su dependencia energética de los países productores de petróleo.

En este sentido, se considera que países como Colombia tienen un gran atraso en torno al aprovechamiento de las fuentes renovables de energía, frente a lo que se convoca a la capacidad emprendedora de los actores sociales para organizarse y generar soluciones, que además de solucionar problemas como el acceso de comunidades alejadas a la electricidad, permitan generar desarrollo local, generando empleo, desarrollo tecnológico y mayor calidad de vida de las poblaciones.

No obstante, el surgimiento de iniciativas emprendedoras está determinado por la existencia de cualidades personales en los actores sociales y un marco institucional propicio, más aún cuando los mercados para las energías renovables no están completamente desarrollados y se requieren de altas inversiones para poner en funcionamiento dichos proyectos. En este sentido, se considera que en Colombia faltan incentivos regulatorios que promuevan este tipo de iniciativas, además de primar un modelo monopolístico en el suministro de energía que limita la introducción de las mismas.

Por tanto, se considera necesario trabajar en la creación de un marco regulatorio que además de estimular el emprendimiento, estimule el desarrollo de tecnologías endógenas y promueva un verdadero desarrollo local en la áreas de impacto de estos proyectos, coordinando los intereses nacionales y privados con los intereses de las comunidades (EPM, 2013). 


\section{REFERENCIAS BIBLIOGRÁFICAS}

Ballenilla, F. (2005). La sostenibilidad desde la perspectiva del agotamiento de los combustibles fósiles, un problema socioambiental relevante. Investigación en la escuela 55. Primer cuatrimestre.

Banco Bilbao Vizcaya Argentaria BBVA (2010). Innovación: perspectivas para el Siglo XXI. Depósito legal: M-515802010. España, p. 1-414.

Boisier, S. (2005). ¿Hay espacio para el desarrollo local en la globalización? Revista de la CEPAL 186.

Burguillo, M. y del Río, P. (2008). La contribución de las energías renovables al desarrollo rural sostenible en la Unión Europea: pautas teóricas para el análisis empírico. Tribuna de Economía ICE, noviembre-diciembre.

Canoura, C. (2010). América Latina ante los efectos irreversibles de un planeta más caliente. Primer Informe Regional sobre Cambio Climático. Tierramérica Medio Ambiente y Desarrollo.

Empresas Públicas de Medellín (EPM) (2013). Energías renovables. Recuperado de: www.epm.con.co.

Formichella, M. (2004). El concepto del emprendimiento y su relación con la educación, el empleo y el desarrollo local. Monografía realizada en el marco de la Beca de Iniciación INTA: "Gestión del emprendimiento y la innovación". Instituto Nacional de Tecnología Agropecuaria, Chacra Experimental Integrada Barrow. Argentina.

Furió, E. (1994). El desarrollo económico endógeno y local: reflexiones sobre su enfoque interpretativo. Estudios Regionales No. 40, pp. $97-112$.

Furlan, A. (2010). Actuar en la crisis: el sistema eléctrico en la perspectiva del desarrollo endógeno. Análisis de caso de la costa atlántica bonaerense, Argentina.

Gallicchio, E. (2004). El desarrollo local en América Latina. Estrategia política basada en la construcción de capital social. Ponencia presentada en el Seminario "Desarrollo con inclusión y equidad: sus implicancias desde lo Local", realizado por SEHAS en la ciudad de Córdoba (Argentina), en mayo de 2004.

Ministerio de Ambiente, Vivienda y Desarrollo Territorial (MAVDT) (2010). Política Nacional de Producción y Consumos Sostenibles.

Páez, A. (2002). La dimensión sociopolítica del fin del petróleo: desafíos a la sostenibilidad. Monografía. 2002. Recuperado de http://www.crisisenergetica.org/staticpages/pdf-rtf/paez_desafios_sostenibilidad-pdf.zip> .

Pérez, J., Sánchez, L., Pardo, M. (2005). La gestión de la demanda de electricidad. Madrid.

Sabogal, J. (2009). La historia se repite: una visión del desarrollo y del desarrollo sostenible. Revista de la Facultad de Ciencias Económicas, XVII, pp. 195-216. 
Sabogal, J. y Moreno, E. (2011). Proyectos forestales de mecanismo de desarrollo Limpio en Colombia: una mirada desde el desarrollo sostenible local. Universidad Militar Nueva Granada.

Sáenz de Miera, G., (20097) “La regulación, clave para el desarrollo de las energías renovables”, en Economía Industrial, 365, 2007, pp. 163-177.

Urquidi, V. (2009). Energías renovables: un enfoque objetivo. Reunión - Diálogo. Expositores, Jorge M. Huacuz y David Nieva. Comentarista Pablo Mulás y Moderador Juan Eibenschutz. Tepoztlán, Morelos, México.

Valdiviezo, G. (2009). Reflexiones en torno a la empresarialidad y el desarrollo económico local. Revista Pueblos y Fronteras Digital, 6, diciembre-mayo 2009, pp. 1- 26.

Vázquez, A. (2009). Desarrollo local, una estrategia para tiempos de crisis. Apuntes del CENES. XXVIII - pp 117 - 132.

Yáñez-Arancibia, A. Day, J. y Hall, C. (2010). Energía, economía y cambio climático: ecuación insoluble. Investigación ambiental 2 (1), pp. 75-82. 